\title{
Early bone healing around implant surfaces treated with variations in the resorbable blasting media method. A study in rabbits
}

\author{
Ryan Jeong ${ }^{1}$, Charles Marin ${ }^{2}$, Rodrigo Granato ${ }^{3}$, Marcelo Suzuki ${ }^{4}$, Jose N Gil ${ }^{5}$, Jose M. Granjeiro ${ }^{6}$, Paulo \\ G. Coelho ${ }^{7}$
}

\footnotetext{
${ }^{1}$ Research Assistant, Dept of Biomaterials and Biomimetics, New York University, New York, USA

${ }^{2}$ Instructor, Dept. of Oral and Maxillofacial Surgery, Pontificia Universidade Catolica do Rio Grande do Sul, Porto Alegre, Brazil

${ }^{3}$ Research Scientist, Department of Dentistry, Oral and Maxillofacial Surgery, Universidade Federal de Santa Catarina, Florianopolis, Brazil

${ }^{4}$ Assistant Professor, Tufts University School of Dental Medicine, Boston, USA

${ }^{5}$ Associate Professor, Department of Dentistry, Oral and Maxillofacial Surgery, Universidade Federal de Santa Catarina, Florianopolis, Brazil

${ }^{6}$ Professor, Department of Cell and Molecular Biology, Universidade Federal Fluminense, Niteroi, Brazil

${ }^{7}$ Assistant Professor, Dept of Biomaterials and Biomimetics, New York University, New York, USA
}

Correspondence:

345 24th street room $813 a$

New York, NY 10010

pgcoelho@nyu.edu

Received: 13/07/2009

Accepted: 02/08/2009

\begin{abstract}
Jeong R, Marin C, Granato R, Suzuki M, Gil JN, Granjeiro JM, Coelho PG. Early bone healing around implant surfaces treated with variations in the resorbable blasting media method. A study in rabbits. Med Oral Patol Oral Cir Bucal. 2010 Jan 1;15 (1):e119-25.

http://www.medicinaoral.com/medoralfree01/v15i1/medoralv15ilp119.pdf
\end{abstract}

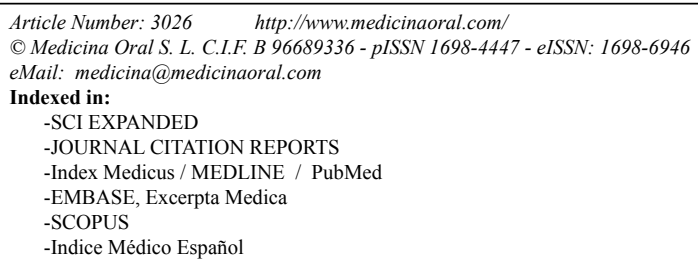

-Indice Médico Español

\begin{abstract}
Objective: this study aimed to histomorphologically and histomorphometrically evaluate the in vivo response to three variations in the resorbable blasting media (RBM) surface processing in a rabbit femur model. Study Design: screw root form implants with $3.75 \mathrm{~mm}$ in diameter by $8 \mathrm{~mm}$ in length presenting four surfaces $(\mathrm{n}=8$ each): alumina-blasted/acid-etched (AB/AE), bioresorbable ceramic blasted (TCP), TCP + acid etching, and AB/AE + TCP were characterized by scanning electron microscopy (SEM) and atomic force microscopy (AFM). The implants were placed at the distal femur of 8 New Zeland rabbits, remaining for 2 weeks in vivo. After sacrifice, the implants were nondecalcified processed to 30 micro $\mathrm{m}$ thickness slides for histomorphology and bone-to-implant contact (BIC) determination. Statistical analysis was performed by one-way ANOVA at $95 \%$ level of significance considering implant surface as the independent variable and BIC as the dependent variable. Results: SEM and AFM showed that all surfaces presented rough textures and that calciu-hosohate particles were observed at the TCP group surface. Histologic evaluation showed intimate interaction between newly formed woven bone and all implant surfaces, demonstrating that all surfaces were biocompatible and osseoconductive. Significant differences in $\mathrm{BIC}$ were observed between the $\mathrm{AB} / \mathrm{AE}$ and the $\mathrm{AB} / \mathrm{AE}+\mathrm{TCP}$, and intermediate values observed for the TCP and TCP + Acid surfaces. Conclusion: irrespective of RBM processing variation, all surfaces were osseoconductive and biocaompatible. The differences in BIC between groups warrant further bone-implant interface biomechanical characterization.
\end{abstract}

Key words: Dental implant, surface treatment, resorbable blasting media, in vivo. 


\section{Introduction}

As the implant surface is the first part of the implant that interacts with the host, methods to increase the implant's biocompatibility and osseoconductivity have been extensively studied (1). Surface modification approaches have been successful in increasing the host response to the surgical implants, resulting in higher bone-to-implant contact (BIC) and biomechanical fixation at early implantation times (2). Modifications in surface texture and chemistry such as increase of roughness, addition of calcium (Ca)- and phosphorous (P)-based bioceramic coatings are the most commonly utilized methods to increase the host response to the surgical implants $(1,2)$. From a surface texture standpoint, increasing surface texture has produced favorable results in both in vitro, in vivo, and ex-vivo studies $(1,2)$. Although more cellular adhesion has been shown for rougher surfaces compared to as-machined or smoother surfaces, microscopically moderately rough surfaces (Ra between 0.5 and $2 \mu \mathrm{m})(1,2)$ have shown better results concerning osseointegration measurable parameters such as BIC and biomechanical testing (3). Surface texturization has been achieved through several processes, which include acid etching, anodization, and grit-blasting with nonresorbable (alumina, silica, titanium oxide) or resorbable blasting media (RBM) such as hydroxiapatite, tricalcium phosphates, or a combination of Ca-P phases (1). It should be noted that most commercially available RBM processes are followed by an acid etching or passivation treatment, resulting in little to absent amounts of $\mathrm{Ca}$ and $\mathrm{P}$ on the final surface $(1,4)$.

From a surface chemistry alteration standpoint, clinical and basic investigations have shown that bioceramiccoated implants such as the thick plasma-sprayed hydroxyapatite (PSHA) present higher host-to-implant response, attaining higher degrees of fixation at earlier implantation times compared to moderately rough uncoated implants (5). PSHA-coated implants, however, rely on mechanical interlocking between the grit-blasted or etched metallic surfaces and the ceramic-like PSHA coating (5). While no evidence supporting that PSHA coated implants presents lower survival rates than uncoated implants, this physical interaction between the bulk metallic, metallic oxide, and bioceramic surface has been considered a weak link in which adhesive failures have reportedly occur on different implant configurations $(5,7)$. Recently, the incorporation of bioactive ceramics on substantially reduced scale has been attempted showing promising results when compared to uncoated textured surfaces (5-7). However, the present literature is inconclusive with respect to the amount and form of $\mathrm{Ca}-\mathrm{P}$ phases results in an optimized early hostto-implant response (4-12).

Since the surface texture and chemistry (especially the amount of $\mathrm{Ca}$ and $\mathrm{P}$ present) of RBM treated surfaces are dependent on several variables such blasting media composition, particle size, processing parameters like blasting pressure and distance, and subsequent acid etching treatments (1), a wide range of surface textures and compositions can be tailored in an attempt to increase the host-to-implant response. However, based on the current literature, no informed design rationale regarding basic RBM processing parameters and associated in vivo response is currently available. Thus, the objective of this study was to histomorphologically and histomorphometrically evaluate the in vivo response to three variations in the RBM surface processing in a rabbit femur model. The surface treatments investigated were: alumina-blasting/acid-etching (AB/AE), alumina-blasting/acid-etching + tricalcium phosphate blasting $(\mathrm{AB} / \mathrm{AE}+\mathrm{TCP})$, tricalcium phosphate blasting (TCP), and tricalcium phosphate blasting + acid-etching (TCP + Acid).

\section{Materials and Methods}

This study utilized screw root form endosseous Ti-6Al-4V implants of $3.75 \mathrm{~mm}$ in diameter by $8 \mathrm{~mm}$ in length (Seven, MIS, Shlomi, Israel) $(n=32)$. The implant groups utilized included the following surfaces: alumina-blasting/acid-etching (AB/AE), alumina-blasting/ acid-etching + tricalcium phosphate blasting ( $\mathrm{AB} / \mathrm{AE}+$ $\mathrm{TCP}$ ), tricalcium phosphate blasting (TCP), and tricalcium phosphate blasting + acid-etching (TCP + Acid). The AB/AE surface treatment was achieved by surface blasting with large particles (size $\sim 300-400$ microns) of A12O3 followed by etching with hydrochloric/sulfuric acid. The TCP surfaces were achieved by blasting with HA/TCP (20/80 percent ratio) particles $200-400$ microns without subsequent acid-etching. The $\mathrm{AB} / \mathrm{AE}+$ TCP surfaces were achieved by combining the aforementioned processes. The TCP + Acid surfaces were achieved by the same process as TCP surface followed by cleaning with HNO3. All implant surfaces were sterilized under gamma radiation.

Surface texturization was accessed by scanning electron microscopy and atomic force microscopy. Scanning electron microscopy (SEM, Philips XL 30, Eindhoven, The Netherlands) was performed at various magnifications under an acceleration voltage of $15 \mathrm{keV}$. Surface three dimensional (3D) imaging were collected by an atomic force microscope (AFM) (Nanoscope IIIa Multimode system, Digital Instruments, Santa Barbara, CA, USA) in contact mode. A scanner with a maximum 125 $\mu \mathrm{m}$ horizontal and $5-\mu \mathrm{m}$ vertical range and a $200-\mu \mathrm{m}$ Si3N4 cantilever tip using a force constant of $0.12 \mathrm{~N} / \mathrm{m}$ was used. The region analyzed was the flat part of the implant cutting edges, and $10 \times 10 \mu \mathrm{m}$ scan areas were used. Three scans were performed per implant.

Following approval of the bioethics committee for animal experimentation at the Universidade Federal Flu- 
minense (\#10/08), Brazil, 8 New Zealand white rabbits weighing between 2.5 and $3 \mathrm{Kg}$ in good health were acquired for the study and were kept in house for 4 weeks prior to the experiment.

The surgical region was the distal femur, and two implants were placed along each limb. The different implant surfaces were alternately placed at proximal and distal sites at distances of $2 \mathrm{~cm}$ from each other along the central region of the bone. The distributions for comparison for the various surfaces resulted in an equal number of implants per group and implantation site (proximal or distal). All surgical procedures were performed under general anesthesia. The pre anesthetic procedure comprised an intra-muscular (IM) administration of ketamin $(20 \mathrm{mg} / \mathrm{Kg})$ and xylasin $(1 \mathrm{mg} / \mathrm{Kg})$. General anesthesia was then obtained following by $1 \%$ isofluorane by inhalation.

Following hair shaving, skin exposure, and antiseptic cleaning with iodine solution at the surgical and surrounding area, a $5 \mathrm{~cm}$ incision at the skin level was performed followed by a muscle layer incision. Then, a flap was reflected and the distal femur exposed.

Two osteotomies per limb were produced at least $2 \mathrm{~cm}$ from each other from distal to proximal. The initial drilling was performed by a $2 \mathrm{~mm}$ diameter pilot drill at $1200 \mathrm{rpm}$ under saline irrigation. Then, sequential drilling with burs of $2.5 \mathrm{~mm}$ and $3.5 \mathrm{~mm}$ was performed at $800 \mathrm{rpm}$ under saline irrigation. The implants were screwed into the osteotomies with a torque wrench. It should be noted that while intimate contact was achieved between bone and the implant microthreads in the cervical region, healing chambers were created due to the interplay between the implant threads' $3.25 \mathrm{~mm}$ inner diameter and the osteotomy $3.5 \mathrm{~mm}$ diameter.

In order to avoid any damage to the implant-bone interface due to removal of a callus overgrowth after limb retrieval, a cover screw was installed in each implant. Standard layered suture techniques were utilized for wound closure (4-0 vicryl- internal layers, 4-0 nylonthe skin). Post-surgical medication included antibiotics (penicillin, 20.000UI/Kg) and analgesics (ketoprophen, $1 \mathrm{ml} / 5 \mathrm{Kg}$ ) for a period of 48 hours post-operatively. The euthanasia was performed by anesthesia overdose.

At necropsy, the limbs were retrieved by sharp dissection, the soft tissue was removed by surgical blades, and initial clinical evaluation was performed to determine implant stability.

Following the necropsy, the bone blocks were kept in $10 \%$ buffered formalin solution for $24 \mathrm{~h}$, washed in running water for $24 \mathrm{~h}$, and gradually dehydrated in a series of alcohol solutions ranging from $70-100 \%$ ethanol. Following dehydration, the samples were embedded in a methacrylate-based resin (Technovit 9100, Heraeus Kulzer GmbH, Wehrheim, Germany) according to the manufacturer's instructions. The blocks were then cut into slices ( $\sim 300 \mu \mathrm{m}$ thickness) aiming the center of the implant along its long axis with a precision diamond saw (Isomet 2000, Buehler Ltd., Lake Bluff, USA), glued to acrylic plates with an acrylate-based cement, and a $24 \mathrm{~h}$ setting time was allowed prior to grinding and polishing. The sections were then reduced to a final thickness of $\sim 30 \mu \mathrm{m}$ by means of a series of $\mathrm{SiC}$ abrasive papers $(400,600,800,1200$ and 2400) (Buehler Ltd., Lake Bluff, IL, USA) in a grinding/polishing machine (Metaserv 3000, Buehler Ltd., Lake Bluff, USA) under water irrigation (13). The sections were then toluidine blue stained and referred to optical microscopy evaluation.

The bone-to-implant contact (BIC) was determined at 50X-200X magnification (Leica DM2500M, Leica Microsystems GmbH, Wetzlar, Germany) by means of a computer software (Leica Application Suite, Leica Microsystems GmbH, Wetzlar, Germany). The regions of bone-to-implant contact along the implant perimeter were subtracted from the total implant perimeter, and calculations were performed to determine the BIC.

Statistical analyses were performed by one-way ANOVA considering implant surface as independent variables and BIC as the dependent variable. The Tukey's post hoc test was used for multiple comparisons. Statistical significance was indicated by p-levels less than $5 \%$.

\section{Results}

Images obtained by SEM showed that all surfaces presented texturization morphologically different (Fig. 1). The TCP and TCP + acid surfaces were qualitatively less rough compared to other two. The TCP surface showed residues of the blasting media particles while $\mathrm{TCP}+$ Acid did not present remnants of blasting media particles (Figure 1b,d). Atomic force microscopy revealed that from a submicrometer standpoint all surfaces were similar (Fig. 2).

The surgical procedures and follow-up demonstrated no complications regarding procedural conditions, post-operative infection, or other clinical concerns. No implants were excluded from the study due to clinical instability immediately after the euthanization.

The nondecalcified sample processing showed intimate bone contact with all implant surfaces at regions of cortical and trabecular bone. Under a magnification of 100X, the bone-implant interfaces were easily visualized and facilitated bone-to-implant contact determination (Fig. 3). In general, woven bone formation occurred in the regions of intimate contact with all tested surfaces. An appositional type bone healing was observed at regions where close contact between implant and bone occurred immediately after implantation, whereas an intramembranous-like bone healing pathway was observed where the interplay of the implant macrogeometry and os- 


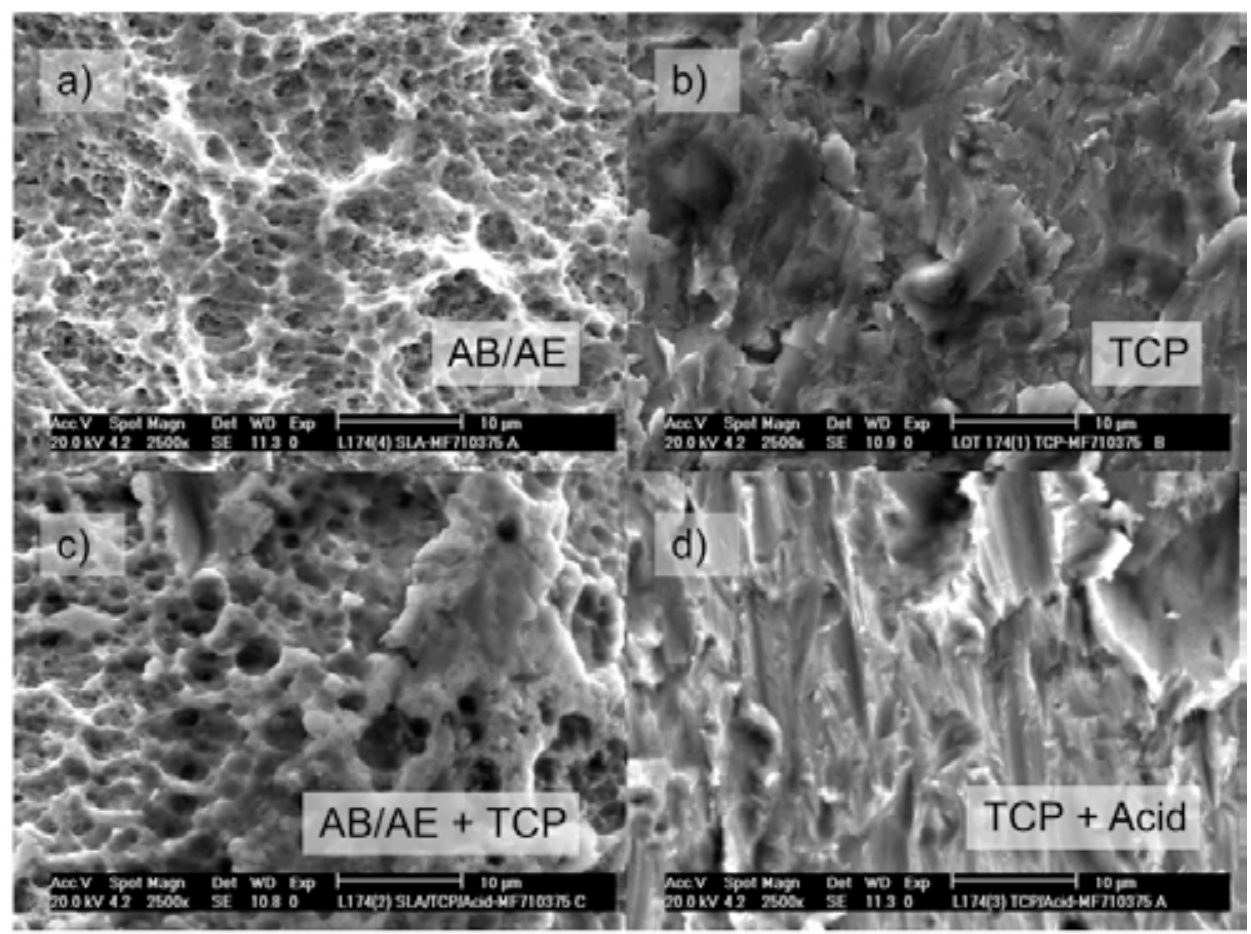

Fig. 1. Scanning electron microscopy images of the a) alumina-blasted/acid-etched (AB/AE), b) TCP, c) AB/ $\mathrm{AE}+\mathrm{TCP}$ and d) TCP + Acid surfaces considered in the present study.
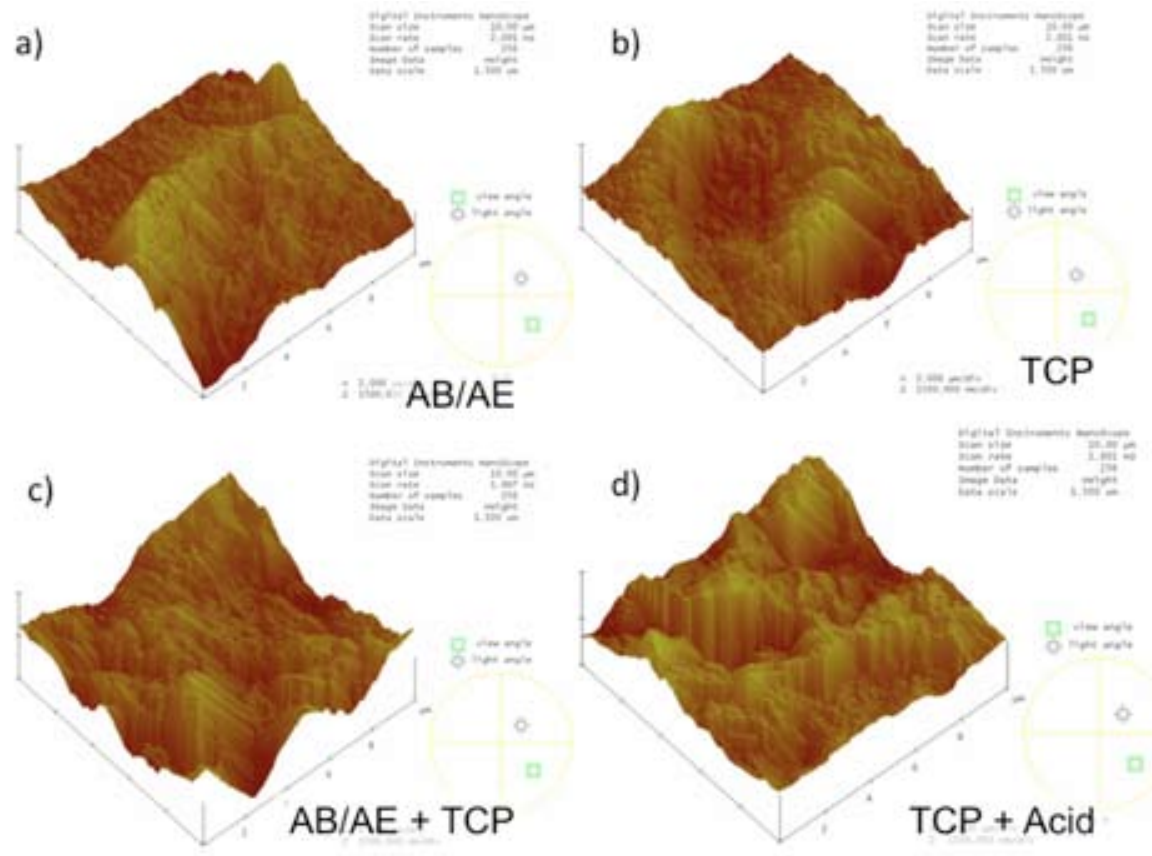

Fig. 2. The three dimensional reconstruction of the different surfaces obtained by AFM showed that at the 10um x 10um scanned area, showed similar roughness patterns for the a) AB/AE b) TCP c) AB/AE + TCP, and d) TCP + Acid surface treatments. 


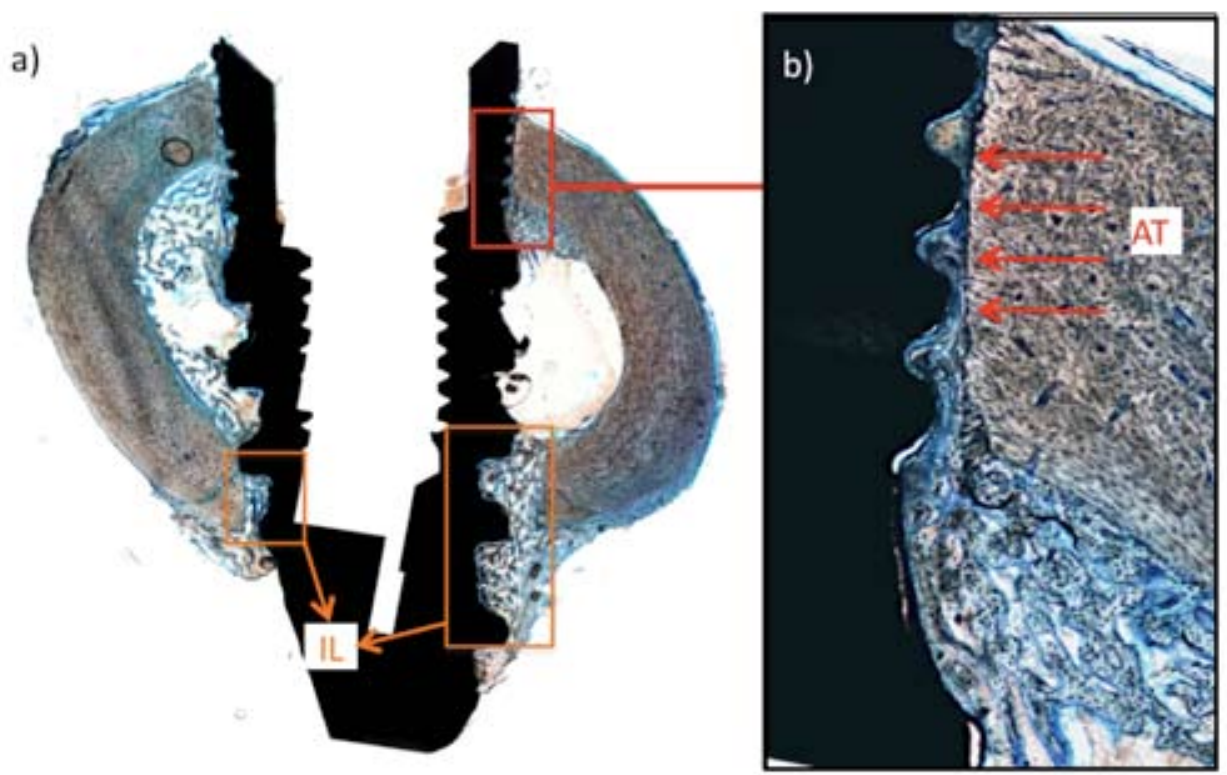

Fig. 3. The bone-implant interfaces were easily visualized and facilitated bone-to-implant contact determination. (a) In general, woven bone formation occurred in intimate contact with all tested surfaces. An apposition type (AT) bone healing was observed at regions where close contact between implant and bone occurred immediately after implantation (b, detail), whereas an intramembranous-like (IL) bone healing pathway was observed where the interplay of the implant macrogeometry and osteotomy dimensions allowed the formation of healing chambers.

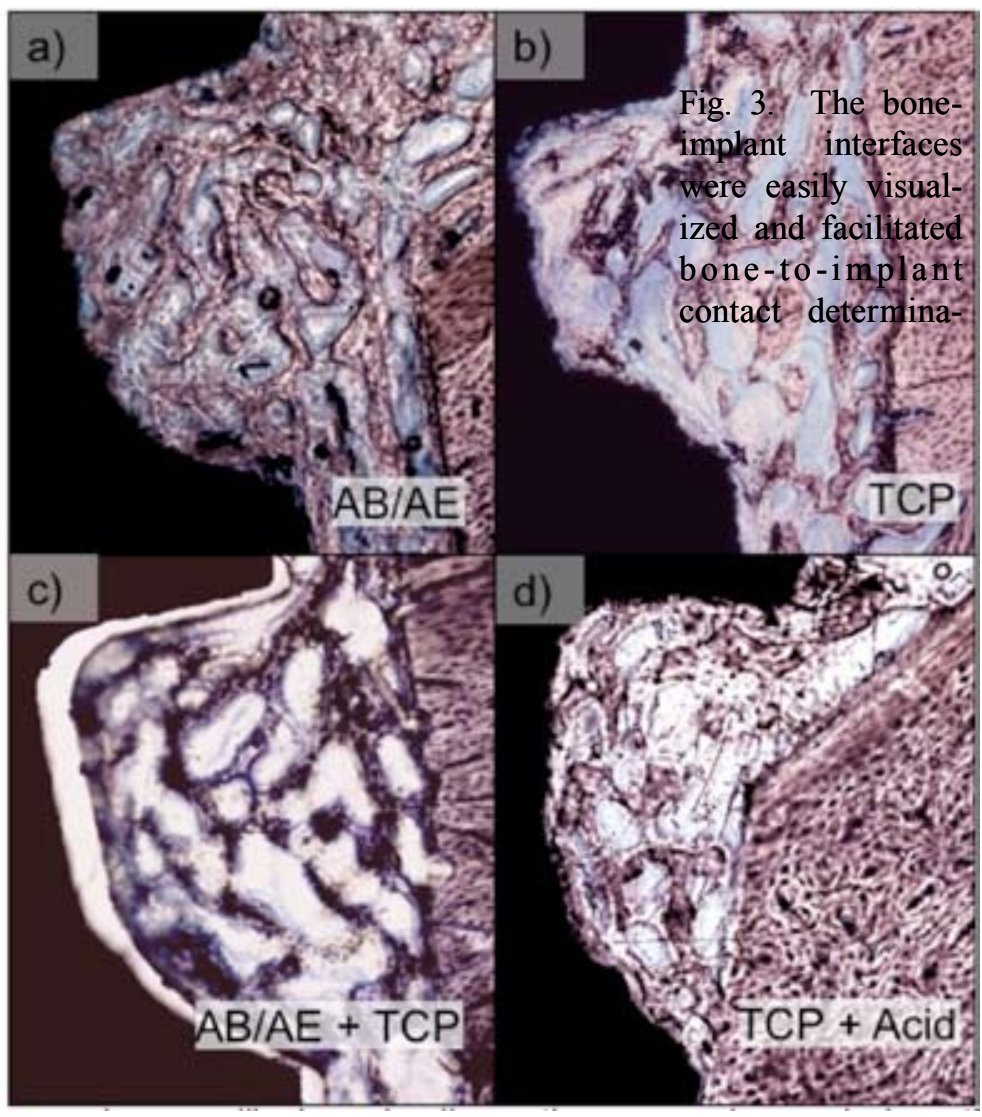

Fig. 4. The intramembranous-like bone healing pathway was observed where the interplay of the implant macrogeometry and osteotomy dimensions allowed the formation of healing chambers. However, no qualitative morphologic differences were observed among the four groups: a) $\mathrm{AB} / \mathrm{AE}$ b) TCP c) $\mathrm{AB} / \mathrm{AE}+\mathrm{TCP}$, and d) TCP + Acid. The gap between implant and bone in (c) is a separation artifact from slide processing. 


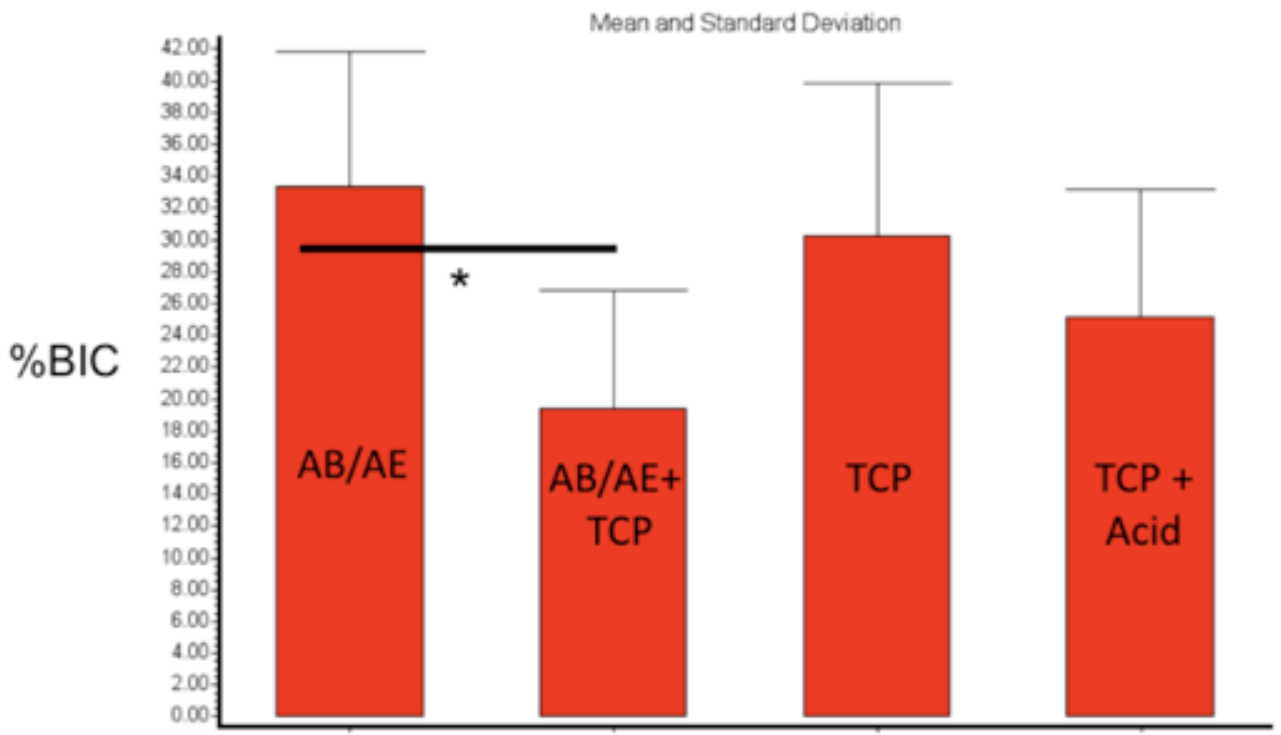

Fig. 5. Mean and standard deviation of \%BIC (bone-to-implant contact). One-way ANOVA showed significant differences between groups $(\mathrm{p}<0.04)$. The asterisk denotes a significant difference between two of the groups $(\mathrm{AB} / \mathrm{AE}$ and $\mathrm{AB} / \mathrm{AE}+\mathrm{TCP})$.

teotomy dimensions allowed the formation of healing chambers (Fig. 3). No significant bone morphological differences were observed between surface groups (Fig. 4). Spatially, woven bone formation occured at central regions and regions in close proximity to the implant surface for all groups, and substantial filling between threads occurred at regions above and below the cortical plate (Fig. 4).

One-way ANOVA showed that the percent bone-to-implant contact was significantly affected by implant surface. The BIC values (expressed as mean \pm standard deviation) for the $A B / A E$ surface were significantly higher than for the AB/AE + TCP surface at $33.38 \pm 8.45 \%$ and $19.37 \pm 7.51 \%$, respectively. The TCP and TCP + Acid surfaces showed intermediate \%BIC values at $30.28 \pm$ $9.60 \%$ and $25.14 \pm 8.07 \%$, respectively (Fig. 5).

\section{Discussion}

Surface modification has been thus far the most investigated design parameter in implant dentistry in an attempt to increase the host-to-implant short- and longterm response $(1,2,14)$. Thus, as wide range of surface modifications have become available under various rationales, and are primarily comprised by roughness and/or chemistry modifications $(1,2,14)$.

The vast majority of the commercially available surfaces present a moderately rough texture ( $\mathrm{Ra}$ values ranging between 0.5 and $2 \mu \mathrm{m})(1,2,14)$ and are typically obtained by a variety blasting and/or acid-etching procedures $(1,2,14)$. Alumina has been thoroughly utilized as a blasting media, however concerns regarding surface cleaning from residual particle embedding prior to sterilization and implantation has led manufacturers into the utilization of alternative biocompatible materials such as titanium oxides and bioactive resorbable ceramics $(1,15)$. While higher biocompatibility degrees are likely achieved by using biocompatible blasting media, especially bioactive ceramics, little is know regarding the effect of tailoring the amount of calcium-phosphates left on the surface through variations in cleaning procedures (primarily acid treatments). Thus, this study aimed to histomorphologically and histomorphometrically evaluate the early in vivo response to three variations in the RBM surface processing in a rabbit femur model.

The four surfaces morphologic characterization revealed texturization in the micrometer and nanometer scale that are commonly observed in commercially available implant surfaces and fall within the moderately rough surface category $(1,15)$. While all surfaces may be considered moderately rough, the differences in the micrometer scale morphology depicted by electron microscopy can be attributed to the different surface processing procedures. For instance, since the resorbable blasting media utilized for the preparation of the TCP and TCP + Acid surfaces presents decreased hardness compared to alumina, smoother roughness profiles were obtained despite the presence and absence of $\mathrm{CaP}$ particles on the TCP and TCP + Acid surfaces, respectively. On the nanometer scale, depicted by AFM, no 
qualitative differences were observed between surfaces. Thus, in the present study, different roughness and surface chemistries were evaluated with respect to its early osseconductivity.

The general histologic findings observed showed that all surfaces investigated were biocompatible and osseoconductive, and all histologic sections showed the natural healing pathway for implants (16-18) irrespective of implant surface. The implant macrogeometry and surgical instrumentation utilized allowed different bone healing patterns that were dependent on how the implant interacted with the final osteotomy (16-18). At regions where intimate contact between bone and implant surface occurred immediately after implant placement, the classic appositional bone healing occurred. At contact-free regions, where healing chambers resulted due to the combination between implant and osteotomy dimensions, woven bone filling was observed through an intramembranous-like healing (16-18). The higher filling between threads observed at regions near the cortical plate occurred due to the higher osteogenic cell content at the periosteal and endosteal regions present in the rabbit femur model. Such characteristic resulted in substantial woven bone formation in close proximity with all surfaces.

Despite the fact that all surfaces were biocompatible and osseoconductive, significant differences in $\mathrm{BIC}$ were observed between the $\mathrm{AB} / \mathrm{AE}$ and the $\mathrm{AB} / \mathrm{AE}+\mathrm{TCP}$, intermediate values observed for the TCP and TCP + Acid surfaces. While controlled physico/chemical characterization protocols must be performed to address this differences, as substantial alteration in surface chemistry may be rendered by the TCP + Acid treatment over the $\mathrm{AB} / \mathrm{AE}$ surface, our results confirm that surface texture or chemistry alone can not be accounted for the early biocompatible properties of implant surfaces. Such observation is further supported by the fact that even though the TCP and TCP + acid surfaces presented similar roughness patterns but different surface chemistry composition. It should be noted that even though the BIC is a commonly utilized measurable indicator of osseointegration parameter, the load bearing capability of the implant may substantially differ as it is dependent in the new ly formed bone mechanical properties and short and long-range implant-bone microstructure may vary substantially (1), warranting further biomechanical characterization (16-18).

\section{References}

1. Coelho PG, Granjeiro JM, Romanos GE, Suzuki M, Silva NR, Cardaropoli $\mathrm{G}$, et al. Basic research methods and current trends of dental implant surfaces. J Biomed Mater Res B Appl Biomater. 2009;88:579-96.

2. Albrektsson T, Wennerberg A. Oral implant surfaces: Part 1-review focusing on topographic and chemical properties of different surfaces and in vivo responses to them. Int $\mathbf{J}$ Prosthodont. 2004;17:536-43.
3. Grizon F, Aguado E, Huré G, Baslé MF, Chappard D. Enhanced bone integration of implants with increased surface roughness: a long term study in the sheep. J Dent. 2002;30:195-203.

4. Marin C, Granato R, Suzuki M, Gil JN, Piattelli A, Coelho PG. Removal torque and histomorphometric evaluation of bioceramic grit-blasted/acid-etched and dual acid-etched implant surfaces: an experimental study in dogs. J Periodontol. 2008;79:1942-9.

5. Coelho PG, Lemons JE. Physico/chemical characterization and in vivo evaluation of nanothickness bioceramic depositions on alumina-blasted/acid-etched Ti-6Al-4V implant surfaces. J Biomed Mater Res A. 2009;90:351-61.

6. Coelho PG, Cardaropoli G, Suzuki M, Lemons JE. Histomorphometric evaluation of a nanothickness bioceramic deposition on endosseous implants: a study in dogs. Clin Implant Dent Relat Res. 2009;11:292-302.

7. Coelho PG, Cardaropoli G, Suzuki M, Lemons JE. Early healing of nanothickness bioceramic coatings on dental implants. An experimental study in dogs. J Biomed Mater Res B Appl Biomater. 2009;88:387-93

8. Mendes VC, Moineddin R, Davies JE. The effect of discrete calcium phosphate nanocrystals on bone-bonding to titanium surfaces. Biomaterials. 2007;28:4748-55.

9. Mendes VC, Moineddin R, Davies JE. Discrete calcium phosphate nanocrystalline deposition enhances osteoconduction on titaniumbased implant surfaces. J Biomed Mater Res A. 2009;90:577-85.

10. Orsini G, Piattelli M, Scarano A, Petrone G, Kenealy J, Piattelli A, et al. Randomized, controlled histologic and histomorphometric evaluation of implants with nanometer-scale calcium phosphate added to the dual acid-etched surface in the human posterior maxilla. J Periodontol. 2007;78:209-18.

11. Wolke JG, Van der Waerden JP, Schaeken HG, Jansen JA. In vivo dissolution behavior of various RF magnetron-sputtered Ca-P coatings on roughened titanium implants. Biomaterials. 2003;24:2623-9. 12. Yang Y, Kim KH, Ong JL. A review on calcium phosphate coatings produced using a sputtering process--an alternative to plasma spraying. Biomaterials. 2005;26:327-37.

13. Donath K, Breuner G. A method for the study of undecalcified bones and teeth with attached soft tissues. The Säge-Schliff (sawing and grinding) technique. J Oral Pathol. 1982;11:318-26.

14. Albrektsson T, Wennerberg A. Oral implant surfaces: Part 2-review focusing on clinical knowledge of different surfaces. Int $\mathrm{J}$ Prosthodont. 2004;17:544-64.

15. Lemons JE. Biomaterials, biomechanics, tissue healing, and immediate-function dental implants. J Oral Implantol. 2004;30:318-24. 16. Berglundh T, Abrahamsson I, Lang NP, Lindhe J. De novo alveolar bone formation adjacent to endosseous implants. Clin Oral Implants Res. 2003;14:251-62.

17. Leonard G, Coelho P, Polyzois I, Stassen L, Claffey N. A study of the bone healing kinetics of plateau versus screw root design titanium dental implants. Clin Oral Implants Res. 2009;20:232-9.

18. Vignoletti F, Johansson C, Albrektsson T, De Sanctis M, San Roman F, Sanz M. Early healing of implants placed into fresh extraction sockets: an experimental study in the beagle dog. De novo bone formation. J Clin Periodontol. 2009;36:265-77.

\section{Acknowledgements}

This study was partially funded by the Dept. of Oral and Maxillofacial Surgery at Universidade Federal de Santa Catarina, NYU summer research program, and MIS implants (Shlomi, Israel). 